VoL. $62(2000) \quad[45-50]$

\title{
THE JULIA SET OF A RANDOM ITERATION SYSTEM
}

\author{
Jı ZHOU
}

This paper presents two properties of the Julia set of a random iteration system of rational functions, which are similar to the well-known results in the classical case.

\section{INTRODUCTION}

Let $\mathcal{R}=\left\{R_{1}, R_{2}, \ldots, R_{M}\right\}$ be a collection of rational functions. In [7], Zhou and Ren first introduced the random iteration system formed by $\mathcal{R}$. Recently, Hinkkanen and Martin [1] developed the rational semigroup generated by $\mathcal{R}$ with the semigroup operation being functional composition. Some properties of their Julia sets have been investigated.

This paper is devoted to developing two properties of the Julia set of the random iteration system, which are similar to the well-known results in the classical case.

Suppose that $Y=\{1,2, \ldots, M\}$ and $\Sigma_{M}=\prod_{0}^{\infty} Y$. A metric $d^{\prime}$ on $\Sigma_{M}$ is given by $d^{\prime}\left(\left\{j_{i}\right\},\left\{j_{i}^{\prime}\right\}\right)=\sum_{i=1}^{\infty}\left|j_{i}-j_{i}^{\prime}\right| / 2^{i}$, where $\left\{j_{i}\right\}$ and $\left\{j_{i}^{\prime}\right\}$ are in $\Sigma_{M}$. For any $\sigma=$ $\left(j_{1}, j_{2}, \ldots, j_{n}, \ldots\right) \in \Sigma_{M}$, we define

$$
W_{\sigma}^{0}(z)=z, \quad W_{\sigma}^{n}(z)=R_{j_{n}} \circ R_{j_{n-1}} \circ \cdots \circ R_{j_{1}}(z),
$$

and its inverse $W_{\sigma}^{-n}(z)$,

$$
W_{\sigma}^{-n}(z)=\left(W_{\sigma}^{n}\right)^{-1}(z)=R_{j_{1}}^{-1} \circ R_{j_{2}}^{-1} \circ \cdots \circ R_{j_{n}}^{-1}(z) . \quad n=1,2, \ldots .
$$

It is known [6] that if $B \subset \Sigma_{M}$, the Julia set $J_{B}(\mathcal{R})$ associated with $B$ is the closure of the union of the sets of non-normality of the sequences $\left\{W_{\sigma}^{n}(z)\right\}$ for all orbits $\sigma$ in $B$. Specifically, we denote by $J(\mathcal{R})$ the Julia set associated with $\Sigma_{M}$ and by $F(\mathcal{R})$ the Fatou set associated with $\Sigma_{M}$, the complement of $J(\mathcal{R})$. (We often call $J(\mathcal{R})$ the Julia set of the random iteration system.) For a random orbit $\sigma \in \Sigma_{M}$, denote by $J(\sigma)$ the Julia set associated with $\sigma$. If $R$ is a rational function, we often denote by $J(R)$ the Julia set of $R$.

Received May 9, 2000

This paper was supported by the Foundation of Zibo Institute. I would like to thank Professor F.Y. Ren for his kind guidance during my study at Fudan University.

Copyright Clearance Centre, Inc. Serial-fee code: 0004-9727/00 \$A2.00+0.00. 
Assume that $z \in J(\mathcal{R})$, and $U$ is a neighbourhood of $z$. Set

$$
E_{U}=\overline{\mathbf{C}}-\bigcup_{\sigma \in \Sigma_{M}} \bigcup_{n>0} W_{\sigma}^{n}(U)
$$

By the Montel theorem, $E_{U}$ contains at most two points. Put $E_{z}=\bigcup_{U} E_{U}$. We call $E_{z}$ the exceptional set of $\mathcal{R}$ at $z$ and each point in $E_{z}$ is called the exceptional point of $\mathcal{R}$. From [7], it is seen that $E_{z}$ is independent of $z$. Hence we omit the subscript $z$ and denote by $E(\mathcal{R})$ the exceptional set of $\mathcal{R}$. Moreover we let $E(R)$ denote the exceptional set of a rational function $R$.

It is well-known that if $R$ is a rational function of degree at least two, and $U$ is any non-empty open set which meets the Julia set $J(R)$ of $R$, then for any compact $V$ which contains no exceptional points of $R$, there exists an integer $l$ such that $R^{n}(U) \supset V$, for $n>l$. For the random iteration system we arrive at

THEOREM 1. Let $J(\mathcal{R})$ denote the Julia set of the random iteration system formed by $\mathcal{R}$ and $E(\mathcal{R})$ be the exceptional set of $\mathcal{R}$. Suppose that $U$ is an open subset of $\overline{\mathbf{C}}$ satisfying $U \cap J(\mathcal{R}) \neq \emptyset$. If $V \subset \overline{\mathbf{C}}$ is a compact set and $V \cap E(\mathcal{R})=\emptyset$, then there exist an open set $N \subset \Sigma_{M}$ and an integer $a>0$ such that for $n>a$ and $\sigma \in N$, $W_{\sigma}^{n}(U) \supset V$.

Let $\overline{\mathbf{C}}$ be the Riemann sphere. By a uniformly perfect set $A$ we mean that $A \subset \overline{\mathbf{C}}$ contains at least one point and there is an $m>0$ such that every annulus that divides $A$ has modulus $\leqslant m$ (see [4]). In [3], Mañé and Da Rocha showed that the Julia set of a rational function is uniformly perfect. Concerning the Julia set of rational semigroup generated by $\mathcal{R}$, a finite set of rational functions, Hinkkanen and Martin [2] also obtained a similar result. Here we consider the Julia set of the random iteration system formed by a finite set of rational functions and obtain the following result using Mañe and Da Rocha's ideas in [3] different from that in [2].

TheOREM 2. Suppose that $J(\mathcal{R})$ denotes the Julia set of the random iteration system formed by $\mathcal{R}$, then $J(\mathcal{R})$ is uniformly perfect.

\section{LEMMAS}

We begin by defining the backward orbit of $\mathcal{R}$ at $z \in \overline{\mathbf{C}}$ as follows:

$$
O^{-}(z, \mathcal{R})=\left\{W_{\sigma}^{-n}(z): \sigma \in \Sigma_{M} \text { and } n \in \mathrm{N}^{+}\right\}
$$

where $\mathrm{N}^{+}$is the set of all positive integers.

We can show that if $z \in E(\mathcal{R})$, then the backward orbit $O^{-}(z, \mathcal{R})$ contains at most two points. Since if $z \in E(\mathcal{R})$, there must be an open set $U$ with $U \cap J(\mathcal{R}) \neq \emptyset$ such that

$$
z \in \overline{\mathbf{C}}-\bigcup_{\sigma \in \Sigma_{M}} \bigcup_{n>0} W_{\sigma}^{n}(U)
$$


or

$$
z \notin \bigcup_{\sigma \in \Sigma_{M}} \bigcup_{n>0} W_{\sigma}^{n}(U)
$$

Hence for any $\zeta \in O^{-}(z, \mathcal{R})$, we have $\zeta \notin \bigcup_{\sigma \in \Sigma_{M}} \bigcup_{n>0} W_{\sigma}^{n}(U)$. Otherwise there are $\sigma_{1}, \sigma_{2} \in$ $\Sigma_{M}$ and $n_{1}, n_{2} \in \mathrm{N}^{+}$such that $W_{\sigma_{1}}^{n_{1}}(\zeta)=z$ and $W_{\sigma_{2}}^{n_{2}}(w)=\zeta$ for some $w \in U$, thus there is $\sigma \in \Sigma_{M}$ such that $W_{\sigma}^{\left(n_{1}+n_{2}\right)}(w)=z$, which is a contradiction. Hence if $z \in E(\mathcal{R})$, we have $\bigcup_{\sigma \in \Sigma_{M}} \bigcup_{n>0} W_{\sigma}^{n}(U) \cap O^{-}(z, \mathcal{R})=\emptyset$. Again since $U \cap J(\mathcal{R}) \neq \emptyset$, the Montel theorem implies that $O^{-}(z, \mathcal{R})$ contains at most two points.

At the same time, if $\mathrm{O}^{-}(z, \mathcal{R})$ contains at most two points, we have $z \in E(\mathcal{R})$. For if it is not true, then $z \in \bigcup_{\sigma \in \Sigma_{M}} \bigcup_{n>0} W_{\sigma}^{n}(U)$, for any open set with $U \cap J(\mathcal{R}) \neq \emptyset$. Now take three mutually disjoint open sets $U_{1}, U_{2}, U_{3}$ with the properties that $U_{i} \cap J(\mathcal{R}) \neq$ $\emptyset, i=1,2,3$. We have $O^{-}(z, \mathcal{R}) \cap U_{i} \neq \emptyset$ and $O^{-}(z, \mathcal{R})$ contains at least three points, which leads to a contradiction.

From the above discussion, we conclude

LEMMA 1. The following statements are equivalent:

(1) $z$ is an exceptional point of $\mathcal{R}$;

(2) the backward orbit $O^{-}(z, \mathcal{R})$ of $\mathcal{R}$ at $z$ contains at most two points.

Using the above lemma, we can obtain the following result.

LEMma 2. Let $\mathcal{R}=\left\{R_{1}, R_{2}, \ldots, R_{M}\right\}$, then

$$
E(\mathcal{R})=\bigcap_{\sigma \in \Sigma_{M}} \bigcap_{n>0} E\left(W_{\sigma}^{n}\right)
$$

where $W_{\sigma}^{n}$ is as defined in (1).

Proof: If $z \in E(\mathcal{R})$, then $O^{-}(z, \mathcal{R})$ contains at most two points. Thus for any $\sigma \in \Sigma_{M}$ and any $n \in \mathrm{N}^{+},\left\{\left(W_{\sigma}^{n}\right)^{-m}(z) \mid m \in \mathrm{N}^{+}\right\}$contains at most two points and $z \in E\left(W_{\sigma}^{n}\right)$ since $z \in E\left(W_{\sigma}^{n}\right)$ if and only if $\left\{\left(W_{\sigma}^{n}\right)^{-m}(z) \mid m \in \mathrm{N}^{+}\right\}$contains at most two points. Thus we have $z \in \bigcap_{\sigma \in \Sigma_{M}} \bigcap_{n>0} E\left(W_{\sigma}^{n}\right)$.

Conversely, if $z \in \bigcap_{\sigma \in \Sigma_{M}} \bigcap_{n>0} E\left(W_{\sigma}^{n}\right)$, but is not in $E(\mathcal{R})$, then for each $\sigma \in \Sigma_{M}$ and any $n \in \mathrm{N}^{+},\left\{\left(W_{\sigma}^{n}\right)^{-m}(z) \mid m>0\right\}$ contains at most two points and $\mathrm{O}^{-}(z, \mathcal{R})$ contains at least two points. Take arbitrarily two points in $O^{-}(z, R)$ different from $z$, say, $z_{i} \in W_{\sigma_{i}}^{-n_{i}}(z), i=1,2$, for some $\sigma_{1}, \sigma_{2} \in \Sigma_{M}$ and some $n_{1}, n_{2} \in \mathrm{N}^{+}$. Since for any $\sigma \in \Sigma_{M}$ and any $n \in \mathrm{N}^{+}, z \in E\left(W_{\sigma}^{n}\right)$, this implies that $z \in\left\{\left(W_{\sigma}^{n} \circ W_{\sigma}^{n}\right)^{-1}(z)\right\}$. Thus we have $\left\{z, z_{1}, z_{2}\right\} \subset\left\{\left(W_{\sigma_{1}}^{n_{1}} \circ W_{\sigma_{1}}^{n_{1}} \circ W_{\sigma_{2}}^{n_{2}} \circ W_{\sigma_{2}}^{n_{2}}\right)^{-1}(z)\right\}$ and $z$ is not an exceptional point of $\left(W_{\sigma_{2}}^{n_{2}} \circ W_{\sigma_{2}}^{n_{2}} \circ W_{\sigma_{1}}^{n_{1}} \circ W_{\sigma_{1}}^{n_{1}}\right)$, a contradiction. Therefore the lemma follows.

To prove our theorems we need the following results.

LEMMA 3. Let $E(\mathcal{R})$ denote the exceptional set of $\mathcal{R}$. 
(1) If $E(\mathcal{R})$ contains only one point, then each $R_{i} \in \mathcal{R}$ is conjugate to a polynomial by the same Möbius transformation.

(2) If $E(\mathcal{R})$ contains two points, then each $R_{i} \in \mathcal{R}$ is conjugate to the map $z \mapsto c_{i} z^{ \pm d_{i}}$ by the same Möbius transformation, where $d_{i}=\operatorname{deg}\left(R_{i}\right)$.

Moreover, all exceptional points lie in $F(\mathcal{R})$.

This is due to Zhou and Ren [7].

Let

$$
\Sigma_{p r d}=\left\{\sigma \in \Sigma_{M}: S^{p}(\sigma)=\sigma, \text { for some } p \in \mathrm{N}^{+}\right\}
$$

where $S$ is the one-sided shift on $\Sigma_{M}$.

Combining [6, Theorem 1] with [5, Theorem 1], we have

LEMMA 4 .

(1) Let $J(\mathcal{R})$ and $J_{\Sigma_{p r d}}(\mathcal{R})$ be the Julia set associated with $\Sigma_{M}$ and the Julia set associated with $\Sigma_{\text {prd }}$ respectively. Then $J(\mathcal{R})=J_{\Sigma_{p r d}}(\mathcal{R})$.

(2) If $\sigma \in \Sigma_{M}$ and for some integer $p, S^{p}(\sigma)=\sigma$, then we have $J(\sigma)=J(g)$ where $g(z)=W_{\sigma}^{p}(z)$ is the characteristic function of $\sigma$.

\section{PROOFS OF THEOREMS}

Proof of Theorem 1: By Lemma 3 we can take the neighbourhood $\mathcal{H}$ of $E(\mathcal{R})$ to be small enough such that for any $R_{i} \in \mathcal{R}, R_{i}(\mathcal{H}) \subset \mathcal{H}$ and $V \subset K=\overline{\mathbf{C}} \backslash \mathcal{H}$ (if $E(\mathcal{R})=\emptyset$, we may assume that $\mathcal{H}=\emptyset$ ). Hence for each $R_{i} \in \mathcal{R}, R_{i}(K) \supset K$. From Lemma 4 it follows that there is an orbit $\sigma_{0}$ and an integer $p$ such that $S^{p}\left(\sigma_{0}\right)=\sigma_{0}$ and $J\left(\sigma_{0}\right) \cap U \neq \emptyset$.

Note that for any rational function $R$ of degree more than one, the exceptional set $E(R)$ contains at most two points. Hence by Lemma 2 there must be three orbits $\sigma_{i} \in \Sigma_{M}, i=1,2,3$, and integers $n_{1}, n_{2}, n_{3}$ such that $E(\mathcal{R})=\bigcap_{i=1}^{3} E\left(W_{\sigma_{i}}^{n_{i}}\right)$.

From Lemma 4, we have $J\left(\sigma_{0}\right)=J(g)$ where $g(z)=W_{\sigma_{0}}^{p}(z)$ is the characteristic function of $\sigma_{0}$. By the classical Fatou-Julia theory, noting that $J\left(W_{\sigma_{i}}^{n_{i}}\right), i=1,2,3$ are perfect, we have that $g^{l}(U)$ for a large enough $l$ covers the Riemann sphere $\overline{\mathbf{C}}$ with the possible exception of (arbitrarily) small neighbourhoods of $E(g)$ and $g^{l}(U) \cap J\left(W_{\sigma_{i}}^{n_{i}}\right) \neq \emptyset, i=1,2,3$. Hence for $l_{1}$ large enough, $\left(W_{\sigma_{1}}^{n_{1}}\right)^{l_{1}} \circ g^{l}(U)$ covers $\overline{\mathbf{C}}$ with the possible exception of (arbitrarily) small neighbourhoods of $E\left(W_{\sigma_{1}}^{n_{1}}\right)$ and for $l_{2}$ large enough, $\left(W_{\sigma_{2}}^{n_{2}}\right)^{l_{2}} \circ\left(W_{\sigma_{1}}^{n_{1}}\right)^{l_{1}} \circ$ $g^{l}(U)$ covers $\overline{\mathbf{C}}$ with the possible exception of (arbitrarily) small neighbourhoods of $E\left(W_{\sigma_{1}}^{n_{1}}\right) \cap E\left(W_{\sigma_{2}}^{n_{2}}\right)$. Thus for $l_{3}$ large enough, $\left(W_{\sigma_{3}}^{n_{3}}\right)^{l_{3}} \circ\left(W_{\sigma_{2}}^{n_{2}}\right)^{l_{2}} \circ\left(W_{\sigma_{1}}^{n_{1}}\right)^{l_{1}} \circ g^{l}(U)$ covers $\overline{\mathbf{C}}$ with the possible exception of (arbitrarily) small neighbourhoods of $E(\mathcal{R})=\bigcap_{i=1}^{3} E\left(W_{\sigma_{i}}^{n_{i}}\right)$ and $\left(W_{\sigma_{3}}^{n_{3}}\right)^{l_{3}} \circ\left(W_{\sigma_{2}}^{n_{2}}\right)^{l_{2}} \circ\left(W_{\sigma_{1}}^{n_{1}}\right)^{l_{1}} \circ g^{l}(U) \supset K$ because $E(\mathcal{R}) \cap K=\emptyset$. Note that for any 
$R_{i} \in \mathcal{R}, R_{i}(K) \supset K$, so we have $R_{i} \circ\left(W_{\sigma_{3}}^{n_{3}}\right)^{l_{3}} \circ\left(W_{\sigma_{2}}^{n_{2}}\right)^{l_{2}} \circ\left(W_{\sigma_{1}}^{n_{1}}\right)^{l_{1}} \circ g^{l}(U) \supset K$. Now choose $a=\sum_{i=1}^{3} n_{i} l_{i}+p l$ and

$$
N=\left\{\sigma \mid \sigma=(\sigma_{0} \mid p l, \overbrace{\left.\sigma_{1}\right|_{n_{1}}, \ldots,\left.\sigma_{1}\right|_{n_{1}}}^{l_{1}} \overbrace{\left.\sigma_{2}\right|_{n_{2}}, \ldots,\left.\sigma_{2}\right|_{n_{2}}}^{l_{2}} \overbrace{\left.\sigma_{3}\right|_{n_{3}}, \ldots,\left.\sigma_{3}\right|_{n_{3}}}^{l_{2}}, j_{a+1}, \ldots)\right\}
$$

where $\left.\sigma\right|_{k}$ denotes the first $k$ elements of $\sigma$ and $j_{n} \in\{1,2, \ldots, M\}$, for $n>a$. Therefore the lemma follows.

Proof of TheOREM 2: Suppose, by way of contradiction, that there is a sequence of annuli $A_{n}, n=1,2, \ldots$, which divide $J(\mathcal{R})$ and satisfy $\lim _{n \rightarrow \infty} \bmod \left(A_{n}\right)=\infty$. This implies that for each $n$, a connected complementary component $K_{n}$ of $A_{n}$ can be chosen so that $\lim _{n \rightarrow \infty} \operatorname{diam} K_{n}=0$. Now denote by $K_{n}^{\prime}$ the other complementary component of $A_{n}$, then $\inf _{n} \operatorname{diam} K_{n}^{\prime}>0$ because otherwise we could take a subsequence $\left\{K_{n_{j}}^{\prime}\right\}$ with $\lim _{j \rightarrow \infty} \operatorname{diam} K_{n_{j}}^{\prime}=0$ and points $p_{j}^{\prime} \in K_{n_{j}}^{\prime} \cap J(\mathcal{R})$ and $p \in K_{n_{j}} \cap J(\mathcal{R})$ converging to points $p^{\prime}$ and $p$ in $J(\mathcal{R})$ respectively, and so, by $\lim _{j \rightarrow \infty} \operatorname{diam} K_{n_{j}}=\lim _{j \rightarrow \infty} \operatorname{diam} K_{n_{j}}^{\prime}=0$ and $J(\mathcal{R}) \subset K_{n_{j}} \cup K_{n_{j}}^{\prime}$ for all $j$, we obtain that $J(\mathcal{R})=p \cup p^{\prime}$. This leads to a contradiction.

Let us denote by $\Delta$ the unit disk and by $\varphi_{n}: \Delta \rightarrow A_{n} \cup K_{n}$ a conformal representation with $\varphi_{n}(0) \in K_{n}$. Then $\bmod \left(\Delta-\varphi_{n}^{-1}\left(K_{n}\right)\right)=\bmod \left(A_{n}\right)$ and $\lim _{n \rightarrow \infty} \operatorname{diam} \varphi_{n}^{-1}\left(K_{n}\right)=0$ because $\lim _{n \rightarrow \infty} \bmod \left(A_{n}\right)=\infty$. Take $1>r_{n}>\rho_{n}>2 \operatorname{diam} \varphi_{n}^{-1}\left(K_{n}\right)$ satisfying $\lim _{n \rightarrow \infty} r_{n}=0$ and $\lim _{n \rightarrow \infty} \rho_{n} / r_{n}=0$. Set $D_{n}=\left\{z|| z \mid<\rho_{n}\right\}$. The family of functions $\varphi_{n}: \Delta \rightarrow \overline{\mathbf{C}}$ is normal because $\inf _{n} \operatorname{diam}\left\{\overline{\mathbf{C}}-\varphi_{n}(\Delta)\right\}=\inf _{n} \operatorname{diam} K_{n}^{\prime}>0$. Hence $\lim _{n \rightarrow \infty} \operatorname{diam} \varphi_{n}\left(D_{n}\right)=$ 0 . But $\varphi_{n}\left(D_{n}\right)$ is an open set containing points of $J(\mathcal{R})$. Thus, from Theorem 1 it follows that there are an integer $k_{n}>0$ and an orbit $\sigma_{n} \in \Sigma_{M}$ such that for $r>$ $k_{n}, W_{\sigma_{n}}^{r}\left(\varphi_{n}\left(D_{n}\right)\right)$ covers $J(\mathcal{R})$. Take $c$ with $0<c<\operatorname{diam} J(\mathcal{R})$ and let $m_{n}$ be the minimal positive integer satisfying $\operatorname{diam} W_{\sigma_{n}}^{m_{n}}\left(\varphi_{n}\left(D_{n}\right)\right) \geqslant c$. Since $\lim _{n \rightarrow \infty} \operatorname{diam} \varphi_{n}\left(D_{n}\right)=0$ and $\mathcal{R}$ is a finite set of rational functions, we obtain that $\lim _{n \rightarrow \infty} m_{n}=+\infty$. Moreover, since $\operatorname{diam} W_{\sigma_{n}}^{m_{n}-1}\left(\varphi_{n}\left(D_{n}\right)\right)<c$, it follows that $\operatorname{diam} W_{\sigma_{n}}^{m_{n}}\left(\varphi_{n}\left(D_{n}\right)\right)<L c$, where $L$ is the maximum of the Lipschitz constants of all $R_{i} \in \mathcal{R}$.

Let $G$ be a set of four different points in $J(\mathcal{R})$ and take $c$ so small that that no set of diameter $\leqslant L c$ contains two of them. Then $W_{\sigma_{n}}^{m_{n}}\left(\varphi_{n}\left(D_{n}\right)\right)$ does not cover three points of $G$. Define $\psi_{n}: \Delta \rightarrow \overline{\mathbf{C}}$ by $\psi_{n}(z)=W_{\sigma_{n}}^{m_{n}}\left(\varphi_{n}\left(r_{n} z\right)\right)$. Next we shall show that the family $\psi_{n}$ is normal. It suffices to show that for all $n, \psi_{n}(\Delta)$ does not cover three points of $G$. If $\rho_{n} / 2 r_{n}<|z|<1$, then $\rho_{n} / 2<\left|r_{n} z\right|<r_{n}$ and, since diam $\varphi_{n}^{-1}\left(K_{n}\right) \leqslant \rho_{n} / 2$ and $0 \in \varphi^{-1}\left(K_{n}\right)$, it follows that $r_{n} z \notin \varphi_{n}^{-1}\left(K_{n}\right)$ and $\varphi_{n}\left(r_{n} z\right) \notin K_{n}$. Hence $\varphi_{n}\left(r_{n} z\right) \notin J(\mathcal{R})$ because $K_{n} \supset J(\mathcal{R}) \cap \varphi_{n}(\Delta)$. Noting that $F(\mathcal{R})$ is forward invariant under $R_{i} \in \mathcal{R}$ (see [7]), we obtain $\psi_{n}(z)=W_{\sigma_{n}}^{m_{n}}\left(\varphi_{n}\left(r_{n} z\right)\right) \in W_{\sigma_{n}}^{m_{n}}(F(\mathcal{R})) \subset F(\mathcal{R})$ and therefore $\psi_{n}(z) \notin G$ when $\rho_{n} / 2 r_{n}<|z|<1$. On the other hand, if $|z| \leqslant \rho_{n} / 2 r_{n}$, it follows that $\left|r_{n} z\right| \leqslant \rho_{n} / 2 \leqslant \rho_{n}$ and then $\psi_{n}(z)=W_{\sigma_{n}}^{m_{n}}\left(\varphi_{n}\left(r_{n} z\right)\right) \in W_{\sigma_{n}}^{m_{n}}\left(\varphi_{n}\left(D_{n}\right)\right)$ that does not 
cover three points of $G$. This proves the normality of the family $\left\{\psi_{n}\right\}$. Then, given $\varepsilon>0$, there is a neighbourhood $N_{0}$ of 0 such that diam $\psi_{n}\left(N_{0}\right) \leqslant \varepsilon$ for all $n$. But for $n$ sufficiently large, $N_{0} \supset\left\{z|| z \mid \leqslant \rho_{n} / r_{n}\right\}$ and

$$
\varepsilon \geqslant \operatorname{diam} \psi_{n}\left(N_{0}\right) \geqslant \operatorname{diam} \psi_{n}\left(\left\{z|| z \mid<\rho_{n} / r_{n}\right\}\right)=\operatorname{diam} W_{\sigma_{n}}^{m_{n}}\left(\varphi_{n}\left(D_{n}\right)\right) \geqslant c .
$$

Since $\varepsilon>0$ is arbitrary, this is a contradiction that proves the theorem.

\section{REFERENCES}

[1] A. Hinkkanen and G.J. Martin, 'The dynamics of semigroups of rational functions I', Proc. London Math. Soc. 73 (1996), 358-384.

[2] A. Hinkkanen and G.J. Martin, 'Julia sets of rational functions', Math. Z. 222 (1996), 161-169.

[3] R. Mañé and L.F. Da Rocha, 'Julia sets are uniformly perfect', Proc. Amer. Math. Soc. 116 (1992), 251-257.

[4] C. Pommerenke, 'Uniformly perfect sets and the Poincaré metric', Arch. Math. 32 (1979), 192-199.

[5] F.Y. Ren, J. Zhou and W.Y. Qiu, 'Dynamics of periodically random orbits', Progr. Natur. Sci. 9 (1999), 248-255.

[6] J. Zhou, W.Y. Qiu and F.Y. Ren, 'Julia sets of random dynamical system and its sub-systems', Chinese Sci. Bull. 43 (1998), 265-268.

[7] W.M. Zhou and F.Y. Ren, A dynamical system formed by a set of rational functions, (H. M. Srivastava and S. Owa, Editors), Current topics in analytic function theory (World Scientific, Singapore, 1992).

Department of Mathematics and Physics

Zibo Institute

Shandong 255013

People's Republic of China 